\title{
Credit Card Fraud Detection Using Machine Learning
}

\author{
Sanobar khan \\ Department of Electronics and \\ Communication \\ Galgotias College Of Engineering and \\ Technology \\ Greater noida, India \\ sanobar2208@gmail.com
}

\author{
Sanovar \\ Department of Electronics and \\ Communication \\ Galgotias College Of Engineering and \\ Technology \\ Greater noida, India \\ sanovarnigam12@gmail.com \\ Mr Hitesh Kumar \\ Department of Electronics and \\ Communication \\ Galgotias College Of Engineering \\ and Technology \\ Greater noida, India \\ hitesh.kumar@galgotiacollege.edu
}

\author{
Suneel Kumar \\ Department of Electronics and \\ Communication \\ Galgotias College Of Engineering \\ and Technology \\ Greater noida, India \\ kumarsuneel2634@gmail.com
}

\begin{abstract}
It is indispensable that credit card organizations can recognize deceitful transactions with the goal that client is not charge for things which they didn't buy. these issues can be handled with data Science and thier significance, alongside machine/soft learning, couldn't be more important. This venture expects to delineate the demonstrating of data sets utilizing machine/soft learning with Credit cards fraud/scam Identification. The Credit Cards fraud/scam detection Issue incorporates displaying previous credit cards exchanges with information of the ones that ended up being misrepresentation. That model is then used for perceive whether another transaction is deceitful or not. Our goal is to recognize $100 \%$ of deceitful transactions and limiting the wrong fraud/scam classification. Credit cards fraud/scam Identification is a average example in grouping. here In this cycle, we had centered on dissecting, pre-preparing datas set collections just lie the sending of numerous inconsistency detection or identification numerous algorithm, for example, Random forest algorithm, KNN algorithm and
\end{abstract}

Keywords - Credit cards fraud/scam application of machine/soft learning, Random forest Algorithms, KNN algorithm,

\section{INTRODUCTION}

'Frauds/scam' for credit cards transaction are unapproved and undesirable utilization for a record from somebody other than the proprietor of that accounts. Essential anticipation estimates could be taken to prevent this maltreatment and conduct of this type of fraudulent acts can concentrated to limit it and ensure in opposition to comparable events in future. In different language, Credit Cards scam can be characterized like a situation in which an individual use another person's credit cards for individual needs while the proprietor and cards giving examined via programmed devices that figure out which transaction for approve.

specialists are ignorant of that way that the card is utilized by someone. scam location includes observing the exercises of populaces of clients to gauge, see or dodge questionable conduct, which comprise of fraud, interruption, and defaulting. This is a pertinent issue that requests the consideration of networks, for example, machine/soft learning and data science in which the answer for this issue can be automate. This issue especially testing by the viewpoint of studying, like it is described from different ways, for example, class imbalances. Quantity of legitimate transaction far dwarf fraud ones. Likewise, transactions designs frequently change its factual characteristics throughout the time. They are not by any means the last difficulties in usage of a genuine scam recognition framework, in any case. In true models, the monstrous stream of instalment demands is immediately examined via programmed devices that figure out which transaction for approve.

Fraud/scam detecting or identification methods is continual prepared to prevent crime person for adopting to their fraud planning. These scams are categorized as: Credit Cards scams: Online and Offline Cards Theft Account Bankrupt gadgets Intrusion solicitation Fraud Counterfeit Card Telecommunication scam. one of recently applied techniques to detect or identify these scams are:

Artificial Neurals Network

Fuzzy Logic Genetic Algorithms

Logistic Regression algorithm

Decision tree algorithm 
Markov Mode techniques

K-Nearest Neighbour

\section{LITERATURE REVIEW}

Real-time Credit Card Fraud/scam Detection Using Machine Learning.[1] This paper centers around four principle fraud events in certifiable transactions. Every fraud is tended to strategy is chosen through an assessment. Significant key territory which we discourse in our venture is constant credit card scam identification.

Credit card scam identification by machine learning techniques: A comparative analysis[2] Data mining had assumed a basic part for identification of credit card scam in payment from internet/online. The exhibition of scam identification in credit card transactions is incredibly influenced from the sampling technique on data sets, determination of factors with detection technique(s) utilized. This research explores the execution of credulous bayes, nearest neighbour with logistic regression on exceptionally skewed credit card scam datas .Credit Card scam identification- Machine Learning methods[3] Credit Card Scam identification database was utilized in an analysis. Since the database was profoundly non balanced, Destroyed strategy was utilized in over sampling. Later on, highlight determination was done and database was part in two sections, preparing data and testing data. The techniques utilized for the investigation were Logistic Regression, Random Forest, Naive Bayes with Multilayer Perceptron..

Credit Card Fraud/scam Detection using Deep Learning[4] This paper is tied in with developing a credit card scam identification framework utilizing Deep Learning Neural Networks. Regardless of whether the Neural Network is prepared above an enormous number of emphases, that isn't adequately accurate to categorize the data as fraud or legitimate due to skewness of the database. We utilize two sampling systems: Under-Sampling, from lessening number of legitimate perceptions and Over-Sampling, in which the fraud class perception is copied. Detection of Credit Card Fraud/scam Transactions Using Machine Learning Algorithms and Neural Networks[5]Credit card fraud coming about because of abuse for the framework is characterized like burglary or abuse of someone's credit card data that is utilized for individual increases unescorted by the consent of the owner of card. For identifying these scams, this is essential for checking the use examples for a client by the previous transaction. Contrasting the utilization example and present day transaction, we could categorize this like one or the other scam or a real transaction. In this research, the procedures utilized are KNN, Naïve Bayes, Logistic Regression, Chebyshev Functional Link Artificial Neural Network (CFLANN), Multi-Layer Perceptron and Decision Trees.

Review on fraud/scam detection methods in credit card transactions[6] Fraud could be admitted from breaking down spend conduct of card owner by previous transaction data. On This publication is licensed under Creative Commons Attribution CC BY. http://dx.doi.org/10.29322/IJSRP.11.06.2021.p11410 the off chance which some digression is shown in spending conduct by reachable samples, may be of fraudulent transaction. For identifying fraud behaviour, banks and credit card departments are using various algorithms of data mining, for samples, choice tree, on the basis of rule mining, neural network, fuzzy bunching technique, hidden Markov technique or mixture approaches of all these techniques. Credit Cards scam identification by Machin/soft Learning and Data Science.[7] This approaches additionally clarified in detailing, how machine learnings could be applied for improve brings about fraud detection alongside the algorithm, pseudocode, clarification its execution and experimentation results. This cycle, we had zeroed in investigating and pre-preparing data set just like organization of numerous oddity identification techniques, for example, Local Outlier Factor, Isolation Forest techniques on PCA changed Credit Cards Transaction information.

Credit Cards scam Detection and Prevention using Machine Learning[8] This exploration zeroed in primarily on distinguishing credit card fraud in genuine world. We should gather the credit card data sets at first for qualified data set. After arbitrary woods algorithm classification technique utilizing the all around assessed data sets and giving current data set. At last, the accurate of outcomes data is streamlined. Credit card fraud/scam detection using Machine learning algorithms.[9]The approach is that we have clarified the idea of frauds identified with credit cards. Here we actualize diverse machine learning algorithms on an imbalanced dataset, for example, logistic regression, naïvebayes, random woodland with troupe classifiers utilizing boosting technique. Different classification models are applied to the data and the model presentation is assessed based on quantitative estimations, for example, accuracy, precision, recall, f1 score, uphold, confusion matrix. The finish of our examination clarifies the best classifier via training and testing utilizing supervised procedures .

Machine Learning For Credit Card Fraud/scam Detection System.[10]It explores the presentation of logistic regression, decision tree with arbitrary timberland for credit card fraud detection. Database of credit cards transactions is gathered by kaggle and it contains an aggregate of 2,84,808 credit card transactions of an European bank data set. Credit Cards Fraud/scam Detection using Machine Learning Algorithms.[11] A principle point of the paper is to plan and build up a novel fraud detection technique for Streaming Transaction Data, with a target, to examine the previous transaction subtleties of the clients and concentrate the conduct patterns. Then utilizing sliding window system to total the transaction made by the cardholders from various gatherings so the standard of conduct of the gatherings can be extricated individually. Later various classifiers are prepared over the gatherings independently. And afterward the classifier with better evaluating score can be picked to be perhaps the best technique to anticipate frauds. Credit Card Fraud/scam Detection: A case study.[12]It explores, the strategy for 'Credit Cards Scam Identification' being progressed. To take care of this issue mix of procedure is utilized such as Genetic Algorithm, Nature build algorithm and Hidden Markov Model. From this transaction is tried exclusively with anyhow goes the finest is additionally 
continued. Presentation checking for class balanced algorithms for credit card scam Detection[13]The presentation of different techniques was assessed utilizing certain exhibition measurements which indicated the proposed approach's productivity. introduced the assessment of the presentation of a few sampling methods on the classifier when these implement on credit cards fraud data set with class imbalance. These key segment investigation (PCA) is applied to genuine data just as the factors time, sum and class to accomplish 28 principal segments that are incorporated.

Deep learning topologies for the detection of fraud/scam in online money transaction.[14] Proposed model beat and forestalled the frauds in any online transaction through credit Cards. Audit on scam identification strategies in credit cards transactions. As indicated by the these parts for scam identification, right around 80 million internet transactions by credit card have been pre-marked like fraud

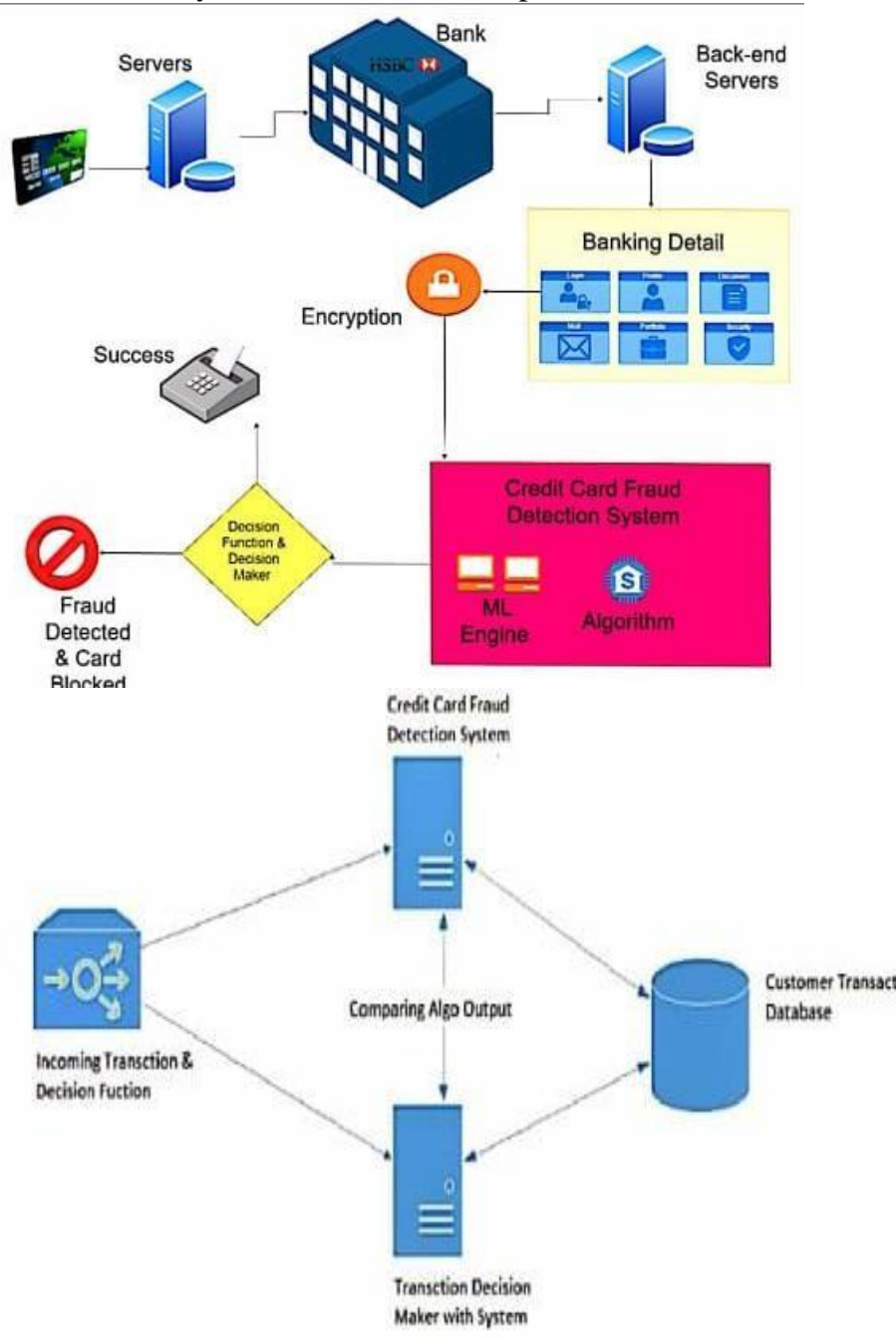

and legitimate. Views on scam identification techniques for credit card transactions [15] The model can be prepared in a more accurate way by adding new features. Several data mining procedures are being utilized by bank and credit card organizations for distinguishing fraud practices. The typical use example of customers relying on their past exercises can be distinguished by applying any of these strategies

\section{METHODOLOGY}

This paper approaches the, use of this new machine/soft learning/soft learning techniques to identify abnormal duties, called outlier. The normal design figure can represent below;

transaction whileb1 says the fraud one. We show different graphical representations to examine conflicts into dataset and to visually comprehend it:

The graphical representation below represent the number of fraud transactions will be more lesser from the valid transaction.

Firstly, we have to obtain the dataset by the website

Kaggle, a data analysist web line that gives data set.

In this the data sets, here are thirty one column and out of 31,28 have given names as v1-v28 to prevent sensitive information. The another columns represent Time, Amount and Class. Time refers to time difference in the first transaction and the next one in row. Amount refers to the money transitioned. Class 0 refers to a legitimate 
Count of Fraudulent vs. Non-Fraudulent Transactions

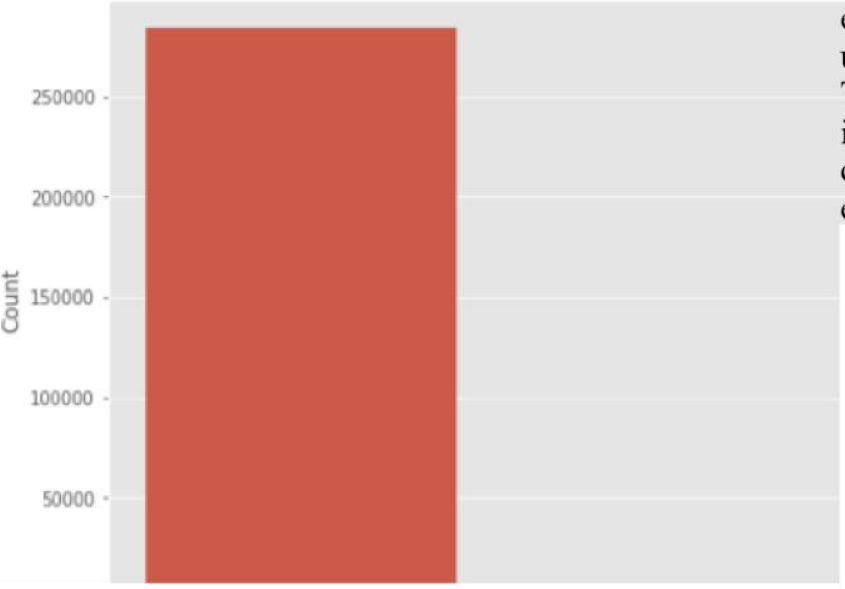

The graphical representation below says the time at that transaction were done in two day. this show the lowest number of transactions which build in night clock and more in the day time.

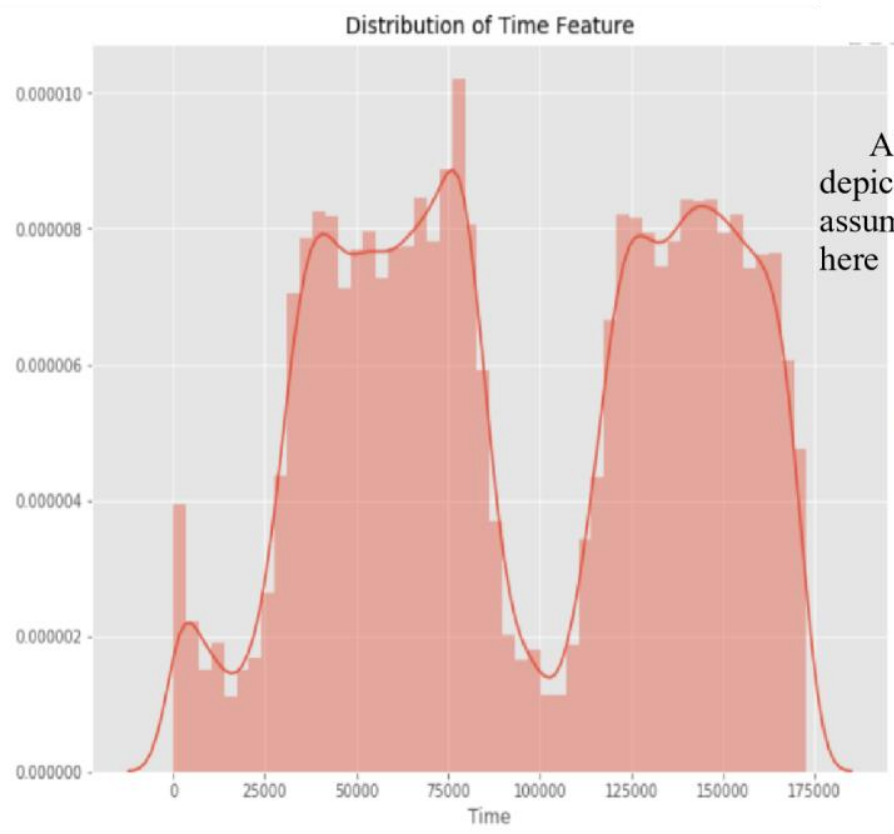

The fig. below shows the amount of money which transact.more of transaction are relative smaller and just a some of this came near to maximum amounts.

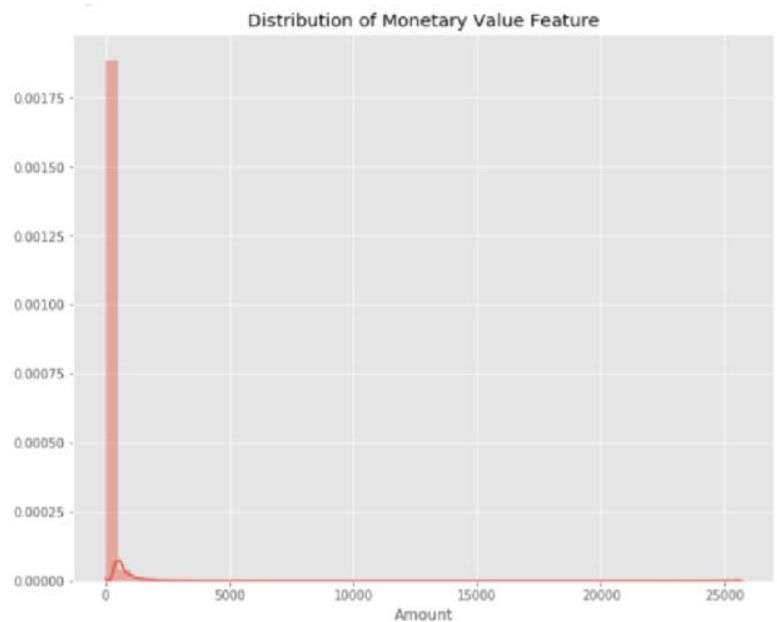

This publication is licensed under Creative Commons Attribution CC BY.

http://dx.doi.org/10.29322/IJSRP.11.06.2021.p11410 here
After analysing this data sets.histogram is being plot for every col. This gives graph of the data sets which could be use to justify that here is no value missed in the data sets. This is to make sure for we do not want any missed values imputation and machines learning/soft learning techniques can proces dataset easily.

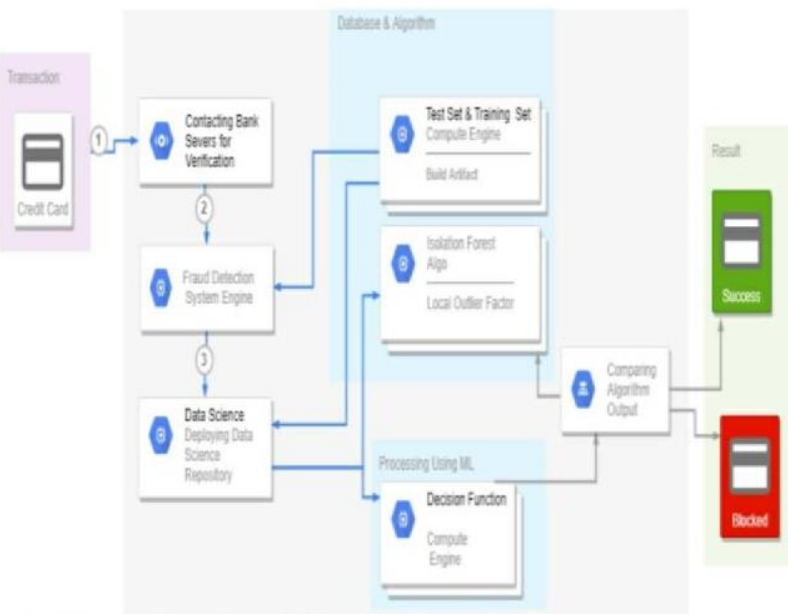

After analization, we graphed a heatmap to have colour depiction for data, for seeing the correlation in output assuming variables and class variables.heatmap represent

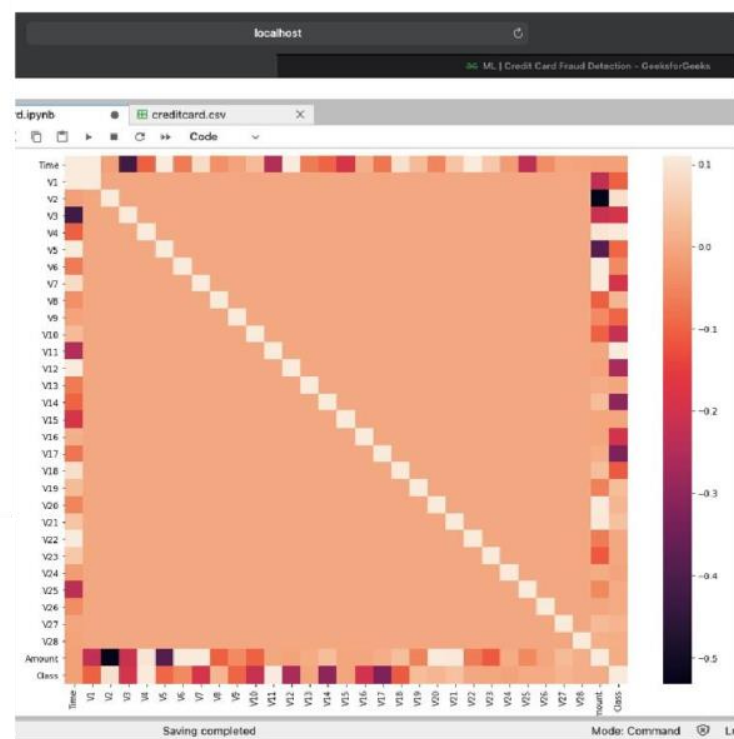

machine learning technique is comes under sklearn. component 
ISSN 2250-3153

in sklearn packing have based on groups technique with functions for the categorization, regression with outlier identification. That is costless with open-source library in python and made by using NumPy, SciPy and matplotlib component that gives a huge of normal with logical devices that could be taken in use by data analysis with soft learning. It has many categorization, clustering with regression techniques and is architecture for interoperate with numerable and scientifically libraries.

The information set is now designed then prepared. The time and amount column were standardized with the Class column will detached to ensure light of assessment. The information is prepared through a set of techniques from modules. The following section structure elaborate how these techniques will come together: This information is fit into a structure and the following detection modules are applied on it:

\section{1- RANDOM FOREST ALGORITHM \\ 2- LOCAL OUTLIER ALGORITHM}

\section{1- RANDOM FOREST ALGORITHM}

Random Forest is additionally known for Random Decision Forest (RFA) that will utilized for categorization, Regression with different assignments which is carried out building numerous decision trees. That Random Forest Algorithm depends onto supervised learning along with significant preferred position for that technique is which that tends to be utilized for categorization and Regression. Random Forest Algorithm has more good accuracy if contrasted and any remaining existing frameworks with that's the most generally utilized technique. This approaches the utilization for Random forest technique in credit card scam identification could show us accuracy of around 90 to $99 \%$.

In credit card scam identification the Random Forest technique show best accuracy into outcomes. Firstly every data sets shall be gathered with investigated. Throughout investigation measure every copy values with furthermore the empty values shall be taken by the dataset. Presently the data sets shall be previously processed dependent onto the amount with dealing/transactions time of discover accuracy for the resultant dataset. Post the pre handling of data sets in money and transaction/dealing period then the data sets shall be isolated into two classifications. The dataset is arranged in two categorization as training data and testing database. Now from data sets categorization, an application software is taken by us known as 'Scikit-learn' software.

Scikit-learn is software which is to machine/soft learning library in python and is free, it has highlights such as classification, regression, Clustering techniques with furthermore different techniques to interoperate along Python. Post the pre preparing of the data sets here now we implement the Random Forest Technique. After the application of Random Forest Technique, the dataset which is pre processed shall be examined once again and a confusion The whole component in sklearn packing have based on groups technique with functions for the categorization, regression with outlier identification. That is costless with open-source library in python and made by using NumPy, SciPy and matplotlib component that gives a huge of normal with logical devices that could be taken in use by data analysis with soft learning. It has many categorization, clustering with regression techniques and is architecture for interoperate with numerable and scientifically libraries.

matrix shall be acquired. Here in confusion matrix the dataset shall be apportioned into four squares as True Positive(TP), False Positive(FP), True Negative(TN)and False Negative(FN). Presently the dataset shall be apportioned consistently till every data will be approved. Presently every apportioned data will be assessed lastly it shall spoken to as isolated graphical representation. Those different graphs will show just low accuracy about the dataset in result. So for getting more accuracy we will utilize Random Forest technique in which it will take every chart value and show just important value along better accuracy when contrasted and any remaining algorithms.

In our designing firstly we take a credit card data set in which it will have insights regarding credit card. Be that as it may, here we set aside just Amount with Transaction effort for examination with pre preparing the dataset. The following stage is the cycle of data cleaning where the database shall be investigated with every copy along empty values shall be killed by the database used. A subsequent stage will be data parting in which the credit card database shall be distributed in two parts like training dataset and test dataset. After the application of Random Forest Technique and a confusion matrix will be gotten. Presently the presentation investigation will be done on the acquired confusion matrix. The production examination shall show accuracy for about $99.9 \%$ of this credit card scam identification framework.

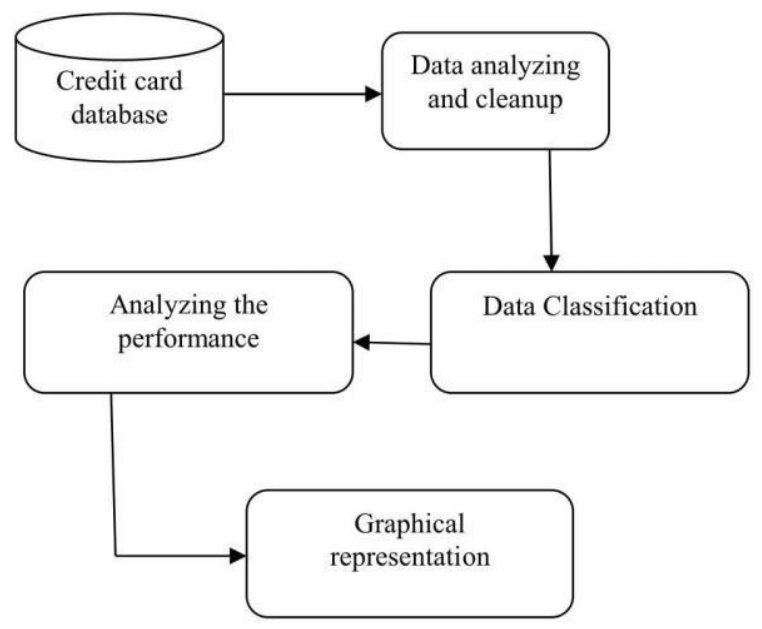




\section{-INVESTIGATIVE DATA ANALYSIS}

Here in this work we initially gather every credit cards datasets then keep it in database. At that point we shall play out a few illustrative investigation about the dataset.

\section{IMPLEMENTATION}

This thought is hard to actualize, all things considered, in light of the fact that it needs the participation by banks, that aren't happy to distribute data because of its bank rivalry, and furthermore because of legitimate causes and insurance data of its client their clients. Subsequently, we saw into research papers that follow comparable methodologies and assembled outcomes. For banking privacy reasons, just synopsis of outcomes got that introduced beneath. Subsequent to applying this procedure, the level 1 rundown envelops a couple of cases yet with a high likelihood of becoming frauds persons. All people referenced in that rundown have its card shut to keep away from some danger because of its heavy-hazard identity. Situation are large mind boggling of them another rundown Another stage rundown are as yet limited satisfactorily to be minded a made to order premise. Credit and assortment officials thought about that a big part for them case for that rundown can be examine like dubious scam conduct. The rearmost rundown including them biggest, job are impartially weighty. Not exactly $33 \%$ of them are dubious. To amplify the time productivity and the over head charge, a chance is to remember another component for the inquiry; this component could be them first numbers of th telephone digits, the email-id, with the secret key, like example, those latest inquiries could be applicable the stage 2 rundown and level 3 rundown.".

\section{-DATA CLEANING}

In the subsequent stage, in the wake of dissecting the datasets afterwards we need to clean-up data. In this cleaning cycle every copy values and empty values which is available in the database shall be eliminated with another dataset shall be acquired.

\section{- PRE TREATING OF DATA SET}

In this module the cleared database shall be pre treated where data set shall be isolated dependent over the amount and transaction time.

\section{- DATASET DISTRIBUTION}

In this module initially the database shall be separated in two parts as training dataset and test database. Right after data parting the Random Forest Technique will pertained. Subsequent to application of Random Forest technique at long last a confusion matrix is gotten.
Presently the resultant data got as confusion matrix could be assessed after utilizing graph portrayal that give more accuracy

\section{2-LOCAL OUTLIER ALGORITHM}

This is a Unsupervised Outlier identification technique. 'Local Outlier Factor' says abnormal score for every example. It calculate the confined difference of trial datas respectively to the adjacent ones. More accurately, placing will be done by k-nearest neighbour, which has displacement use for measures the local data .

\section{RESULT}

The code inscribe quantity of false positives, identified furthermore, contrasts it and the true values. That is utilized to estimate the accuracy score and precision for the

techniques. That part for data we have utilized by quicker examine is $10 \%$ out of the whole data sets. The total dataset is likewise utilized toward the last and all the outcomes are inscribed. That result and the categorization result by every technique being taken into the yield like follows, in which class 0 methods the transactions is resolved to be legitimate including 1 methods that is resolved as an scam transactions. The below outcome coordinated opposed to the class values to test for fake certainties . Results if full dataset being used: 

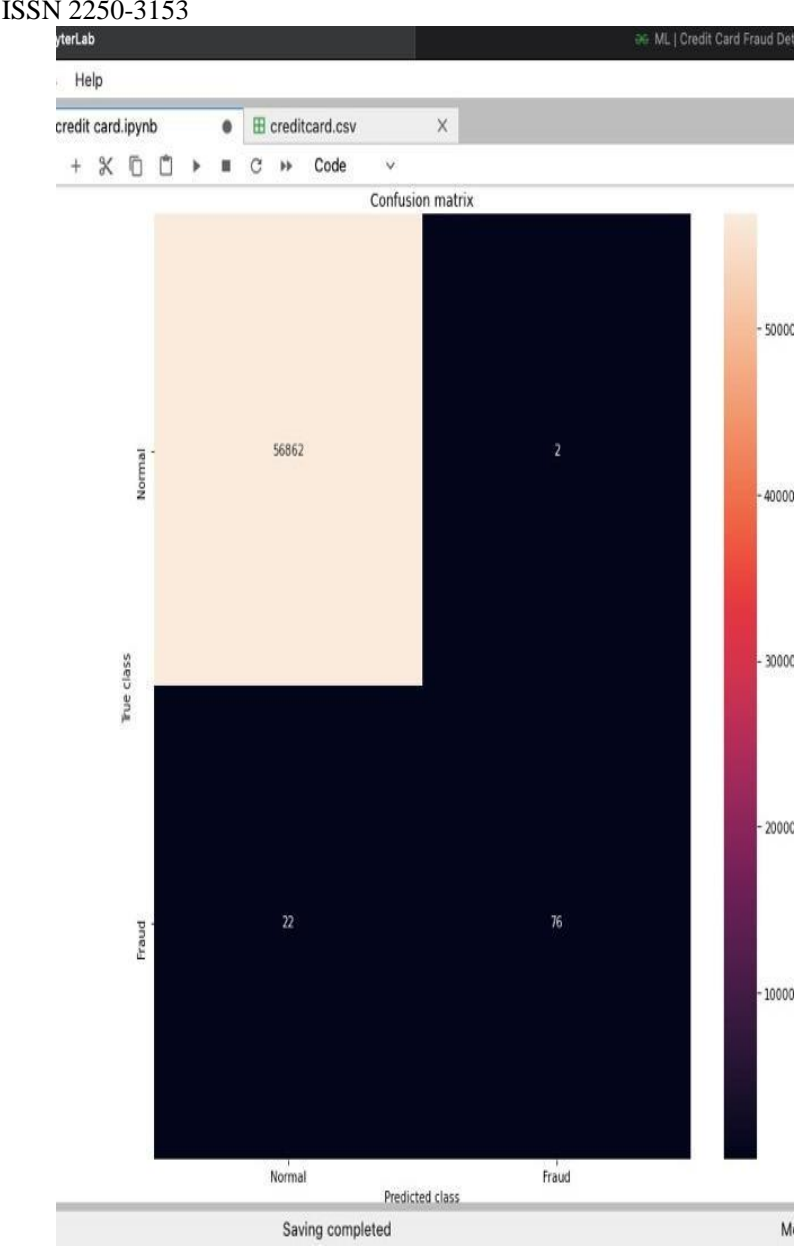

Result of Random Forest:-

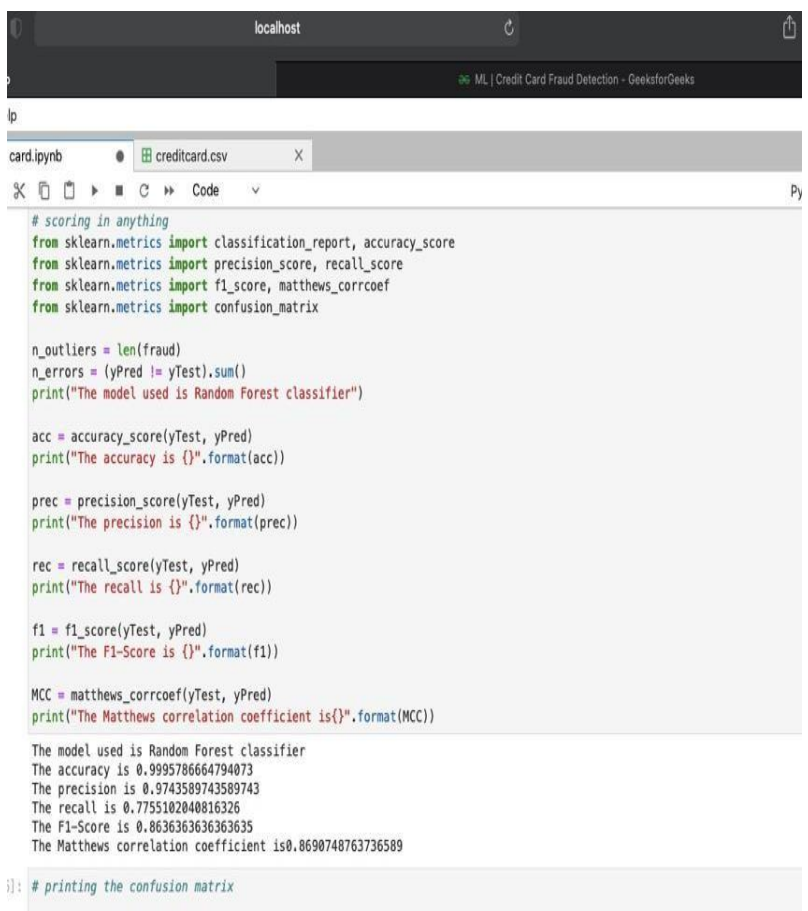

Result of local outlier algorithm:-
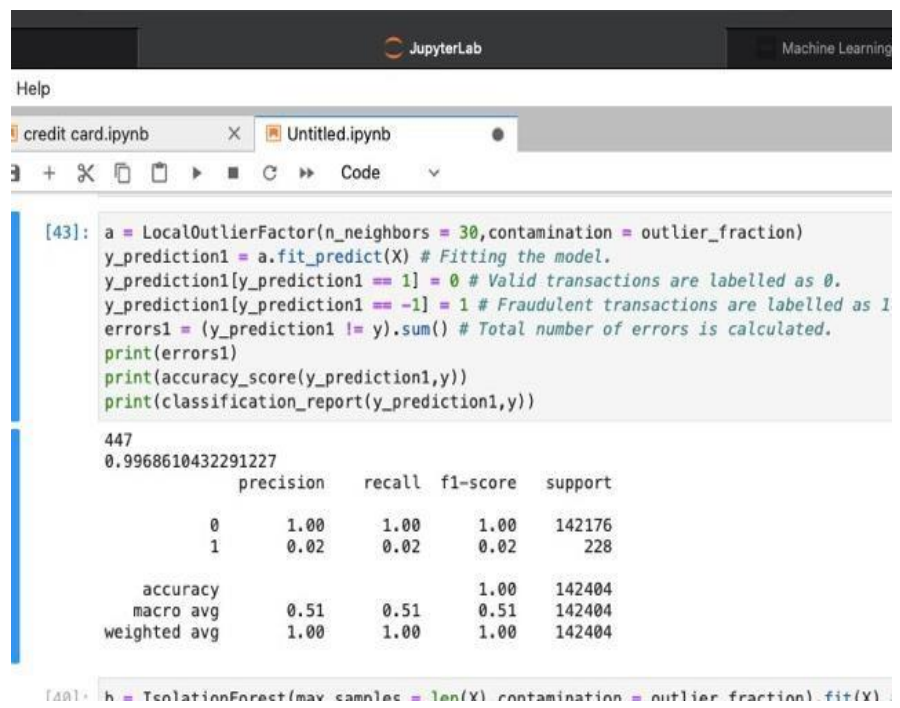

\section{CONCLUSION}

Credit card scam is indeed as demonstration of delinquent deceit. The paper have drilled down the nearly widely recognized techniques for scam alongside their detection strategies and looked into late discoveries in the meadow. The article have additionally clarified in attribute, how artificial intelligence could be appealed with improve brings about scam detection alongside the algo, pseudocoel, clarification it usage and testing upshot. Though the algo comes to approx $99.7 \%$, its exactness stays just at $29 \%$ when the 10th of this dataset is contemplated. Nonetheless, when the whole dataset is taken care of in this algo, the exactness ascends to $34 \%$. That big level of fidelity are to be relied upon because of the tremendous disparity among the quantity of substantial and count of certifiable dealings. As observed this whole data set have just 2 day transactions results, their only an portion of data which could have made accessible if the protude was to be utilized in the mercantile measure. It depends on artificial intelligence techniques, this code shall just extend their productivity of tempo as most datas are vent onto this.

\section{FUTURE ENHANCEMENT}

When we can not reach out objective for $100 \%$ accuracy in scam identification, we wound up making a framework which could, along sufficient opportunity with data, get near this objective. Similarly like along any venture, here are such opportunity to get better here. The very nature of this undertaking takes into account various techniques for to be coordinated jointly as blocks with its outcomes could be joined for building them accuracy for end product. That work can additionally be enhanced along with the option of many techniques in them. Be that as it may, the yield of these algorithms should be in a similar configuration as the another. When this situation are fulfilled, these module, is anything but difficult to plus like crisp in the code. That gives an incredible level of particularity and flexibility to the project. More opportunity to get better can be found in the dataset. As shown previously, the precision of the algorithms increments . 
[1] Anuruddha Thennakoon; Chee Bhagyani; Sasitha Premadasa; Shalitha Mihiranga; Nuwan Kuruwitaarachchi 2019 9th International Conference on Cloud Computing,

Data Science \& Engineering (Confluence);10-11 Jan. 2019

[2]John O. Awoyemi; Adebayo O. Adetunmbi; Samuel A. Oluwadare; 2017 International Conference on Computing Networking and Informatics (ICCNI) 29-31 Oct. 2017.

[3]Dejan Varmedja; Mirjana Karanovic; Srdjan Sladojevic; Marko Arsenovic; Andras Anderla ; 2019 18th International Symposium INFOTEH-JAHORINA (INFOTEH) 20-22 March 2019

[4]Pranali Shenvi; Neel Samant; Shubham Kumar; Vaishali Kulkarni ; 2019 IEEE 5th International Conference for Convergence in Technology (I2CT) 29-31 March 2019

[5] Deepti Dighe; Sneha Patil; Shrikant Kokate ; 2018 Fourth International Conference on Computing Communication Control and Automation (ICCUBEA)16-18 Aug. 2018

[6] Krishna Modi; Reshma Dayma; 2017 International Conference on Intelligent Computing and Control (I2C2); 23-24 June 2017

[7] S P Maniraj;Aditya Saini;Shadab Ahmed ; International Journal of Engineering and Technical Research 08(09); September 2019; vol 08;page no. 110-115.

[8] S. Abinayaa, H. Sangeetha, R. A. Karthikeyan, K. Saran Sriram, D. Piyush; International Journal of Engineering and Advanced Technology (IJEAT) ;4, April, 2020;vol 09; page no. 1199-1201.

[9] K.Ratna Sree Valli , P.Jyothi , G.Varun Sai , R.Rohith Sai Subash; Quest Journals Journal of Research in Humanities and Social Science; 2 June 2019; Volume 8; page no: 04-11

[10] Lakshmi S V ; Selvani Deepthi Kavila; International Journal of Applied Engineering Research ISSN; 04 November 2018; Volume 13, pp. 16819-16824.

[11] Vaishnavi Nath Dornadulaa; Geetha S; INTERNATIONAL CONFERENCE ON RECENT TRENDS IN ADVANCED
COMPUTING 2019, ICRTAC 2019; December 2019; vol 08; page no. 631-641.

[12] Ayushi Agrawal; Shiv Kumar; Amit Kumar Mishra; 20152 nd Internation al Conference on Computing for Sustainable Global Development (INDIACom); 11-13 March 2015; IEEE ; vol 09; page 231-242

[13] D. S. Sisodia, N. K. Reddy and S. Bhandari; IEEE International Conference on Power, Control, Signals and Instrumentation Engineering (ICPCSI),2017; Chennai, pp.2747-2752.

[14] A. Roy and J. Sun and R. Mahoney and L. Alonzi and S. Adams and P. Beling, "Deep learning detecting fraud in credit card transactions," IEEE International Conference on in Systems and Information Engineering Design Symposium(SIEDS), pp. 129-134, 2018.

[15] K. Modi and R. Dayma, "Review on fraud detection methods in credit card transactions, "International Conference on Intelligent Computing and Control (I2C2), Coimbatore, pp. $1-5,2017$. 